\title{
Numerical Approximation of Fractional-Order Volterra Integrodifferential Equation
}

\author{
Xiaoli Qiang, ${ }^{1}$ Kamran $\mathbb{D}^{2},{ }^{2}$ Abid Mahboob, ${ }^{3}$ and Yu-Ming Chu $\mathbb{D}^{4,5}$ \\ ${ }^{1}$ Institute of Computing Science and Technology, Guangzhou University, Guangzhou 510006, China \\ ${ }^{2}$ Department of Mathematics, Islamia College Peshawar, Khyber Pakhtoon Khwa, Pakistan \\ ${ }^{3}$ Department of Mathematics, Division of Science and Technology, University of Education, Lahore, Pakistan \\ ${ }^{4}$ Department of Mathematics, Huzhou University, Huzhou 313000, China \\ ${ }^{5}$ Hunan Provincial Key Laboratory of Mathematical Modeling and Analysis in Engineering, Changsha University of Science \\ \& Technology, Changsha 410114, China
}

Correspondence should be addressed to Yu-Ming Chu; chuyuming@zjhu.edu.cn

Received 21 September 2020; Revised 27 October 2020; Accepted 26 November 2020; Published 16 December 2020

Academic Editor: Jiabin Zuo

Copyright ( 2020 Xiaoli Qiang et al. This is an open access article distributed under the Creative Commons Attribution License, which permits unrestricted use, distribution, and reproduction in any medium, provided the original work is properly cited.

\begin{abstract}
Laplace transform is a powerful tool for solving differential and integrodifferential equations in engineering sciences. The use of Laplace transform for the solution of differential or integrodifferential equations sometimes leads to the solutions in the Laplace domain that cannot be inverted to the real domain by analytic methods. Therefore, we need numerical methods to invert the solution to the real domain. In this work, we construct numerical schemes based on Laplace transform for the solution of fractional-order Volterra integrodifferential equations in the sense of the Atangana-Baleanu Caputo derivative. We propose two numerical methods for approximating the solution of fractional-order linear and nonlinear Volterra integrodifferential equations. In our scheme, the inverse Laplace transform is approximated using a contour integration method and Stehfest method. Some numerical experiments are performed to check the accuracy and efficiency of the methods. The results obtained using these methods are compared.
\end{abstract}

\section{Introduction}

Fractional calculus has a large number of applications in engineering and mathematical sciences [1-5]. Problems from engineering and other sciences which involve derivatives or integrals of noninteger orders are large in number and still growing. That is why the research community has showed great interest in the area of fractional calculus. One can find various applications of fractional calculus in numerous phenomena such as frequency-dependent damping behavior of viscoelastic materials [6], control theory [7], economics [8], mass and heat diffusion processes, electromagnetic theory, biological species [9], robotics, and many other engineering problems [10]. Accurate models of systems under consideration can be obtained using fractional differential and integrodifferential equations [11]. Many remarkable works on fractional calculus are available in literature for the approxi- mation of the solution fractional differential or integrodifferential equations, for example, the sinc-collocation method [12], Legendre collocation method [13], Laguerre polynomials [14], Adomian decomposition method [15], Variational iteration method [16-18], and their references.

Numerous essential phenomena in nature are resolved using the solutions of fractional integrodifferential equations $[11,19]$. For example, one can find applications of fractional integrodifferential equations (FIDEs) in electromagnetics [20], heat conduction [21], etc. Due to the wide range of applications, FIDEs have attracted researchers. Therefore, a large number of analytic and numerical methods have been developed for finding the solutions of FIDEs [22-26]. For example, the authors [27] utilized the fractional differential transform method for the solution of fractional integrodifferential equations. A Legendre wavelet method [28] is proposed for the solution of FIDEs. In [29], the analytic 
solution of FIDEs is obtained using the homotopy analysis method. The author in [30] obtained the analytic solution of FIDEs using the fractional residual power series method.

In many cases, the analytic solutions of fractional differential or integrodifferential equations are hard to obtain, so numerical methods must be used. In this connection, the researchers have developed numerous numerical methods. For instance, the authors in $[19,31]$ have solved FIDEs using the reproducing kernel Hilbert space method. A hybrid collocation method [32] is proposed to solve the FIDEs. The authors in [33] solved the first-order Volterra equation by the collocation method. The author in [34] utilized the shifted Chebyshev polynomial and least squares method for the approximation of the solution of FIDEs. Mahdy and Shwayyea [35] obtained the approximate solution of FIDEs using the shifted Laguerre polynomial pseudospectral method and least squares method. The authors in [36] approximated the solution of Volterra-type FIDEs via Bernoulli wavelet approximation. In [14], Laguerre polynomials are utilized to approximate FIDEs of Volterra type. More work on the solution of FIDEs can be found in [31, 33, 37, $38]$ and their references.

There are various definitions of fractional derivatives such as Caputo-Liouville's and Riemann-Liouville's [11, 39]. These derivatives have certain disadvantages due to nonlocal and nonsingular kernel functions involved in these derivatives. In order to avoid these difficulties, it is better to use the $\mathrm{ABC}$ derivative. The $\mathrm{ABC}$ derivative contains a nonsingular kernel and therefore can model a phenomenon which cannot be handled by fractional-order derivatives having singular kernels [40]. Some recent articles on the application of the $\mathrm{ABC}$ derivative can be found in References [4145]. In the present work, we consider a FIDEs of Volterra type with the $\mathrm{ABC}$ derivative of the form

$$
\begin{gathered}
{ }_{0}^{\mathrm{ABC}} D_{t}^{\beta} \zeta(t)=\mathscr{H}(t, \zeta(t), \mathscr{T} \zeta(t)), \\
\mathscr{T} \zeta(t)=\int_{0}^{t} \mathscr{B}(t, \tau) \mathscr{Q}(\zeta(t)) d \tau, \\
\zeta(0)=\alpha,
\end{gathered}
$$

where $\beta \in(0,1], 0 \leq t, \tau \leq 1, \alpha \in \mathbb{R}, \mathscr{H}, \mathscr{B}, \mathscr{Q} \in C[0,1]$.

Here, ${ }_{0}^{\mathrm{ABC}} D_{t}^{\beta}$ is the $\mathrm{ABC}$ derivative of order $\beta$.

\subsection{Preliminaries}

Definition 1. The ABC fractional derivative of order $0<\beta<1$, of $\zeta \in \mathcal{S}^{1}(a, b)$ with base point $a$ at $t \in(a, b)$, is defined as [46]

$$
{ }_{0}^{\mathrm{ABC}} D_{t}^{\beta} \zeta(t)=\frac{\mathscr{M}(\beta)}{1-\beta} \int_{a}^{t} \zeta^{\prime}(s) E_{\beta}\left[\frac{-\beta}{1-\beta}(t-s)^{\beta}\right] d s,
$$

where $\delta^{1}$ is a Sobolev-space of first-order fitted with $L^{2}$ -norm defined over the $\Omega \subset \mathbb{R}$ defined as follows:

$$
\mathcal{S}^{1}(\Omega)=\left\{\zeta \in L^{2}(\Omega): \zeta^{\prime} \in L^{2}(\Omega)\right\}
$$

where $\mathscr{M}(\beta)$ is defined as follows:

$$
\mathscr{M}(\beta)=\frac{\beta}{\Gamma(\beta)}+1-\beta
$$

also, $E_{\beta}(t)$ is a one-parameter Mittage-Leffler (ML) function defined as

$$
E_{\beta}(t)=\sum_{m=0}^{\infty} \frac{t^{m}}{\Gamma(m \beta+1)}, \quad \beta>0,-\infty<t<\infty
$$

Definition 2. The $\mathrm{ABC}$ fractional integral of order $0<\beta<1$ of $\zeta \in \mathcal{S}^{1}(a, b)$ with base point $a$ at $t \in(a, b)$ is defined as [46]

$$
\mathcal{F}_{t}^{\beta} \zeta(t)=\frac{1-\beta}{\mathscr{M}(\beta)} \zeta(t)+\frac{\beta}{\mathscr{M}(\beta) \Gamma(\beta)} \int_{a}^{t} \zeta(s)(t-s)^{\beta-1} d s .
$$

Definition 3. The Laplace transform of a piecewise continues function $\zeta(t)$ is defined as

$$
\mathscr{L}\{\zeta(t)\}=\widehat{\zeta}(s)=\int_{0}^{\infty} e^{-s t} \zeta(t) d t .
$$

Definition 4. If $0<\beta \leq 1$, then the Laplace transform of the $\mathrm{ABC}$ derivative is defined by

$$
\mathscr{L}\left\{{ }_{0}^{\mathrm{ABC}} D_{t}^{\beta} \zeta(t)\right\}=\frac{\mathscr{M}(\beta) s^{\beta} \widehat{\zeta}(s)-\mathscr{M}(\beta) s^{\beta-1} \zeta(0)}{s^{\beta}(1-\beta)+\beta} .
$$

\section{Proposed Method}

We provide a description of our proposed schemes. Our numerical scheme has three main steps. In our first step, we apply the Laplace transform to the given model, with which the problem is reduced to an algebraic equation. In the second step, we solve the reduced equation in Laplace space for the unknown. Finally, the solution of the original problem is obtained using numerical inversion of Laplace transform. In this work, the numerical approximation of Laplace transform is obtained using two schemes. One is the contour integration method (CIM), and second is the Stehfest method (SM). We discuss these methods in the next sections. First, we apply Laplace transform to Eq. (1); we get

$$
\frac{\mathscr{M}(\beta) s^{\beta} \widehat{\zeta}(s)-\mathscr{M}(\beta) s^{\beta-1} \zeta(0)}{s^{\beta}+\left(1-s^{\beta}\right) \beta}=\widehat{\mathscr{H}},
$$

which can be rearranged as

$$
\widehat{\zeta}(s)=\frac{\widehat{\mathscr{G}}(s)}{s^{\beta} \mathscr{M}(\beta)},
$$

where

$$
\widehat{\mathscr{G}}(s)=\mathscr{M}(\beta) s^{\beta-1} \alpha+\widehat{\mathscr{H}}\left(s^{\beta}\left(1-s^{\beta}\right) \beta\right) .
$$


In order to obtain the solution of problem (1), we apply Laplace inverse on (12), and the Laplace inverse is then approximated using the contour integration method and the Stehfest method, which are discussed as follows.

\section{Contour Integration Method (CIM)}

In CIM, we represent the solution $\zeta(t)$ of (1) as Bromwich integral:

$$
\zeta(t)=\frac{1}{2 \pi \iota} \int_{\Gamma} e^{s t} \widehat{\zeta}(s) d s, \text { Res } \geq \vartheta
$$

where $\vartheta \in R$ and $\Gamma$ is an initially appropriately chosen line $\Gamma_{0}$ in a complex plane parallel to the $y$ axis, with Ims $\rightarrow \pm \infty$. Then, in (14), $\zeta(t)$ is the inverse transform of $\zeta_{b}(s)$, satisfying the condition that all the singularities of $\zeta_{b}(s)$ lie to the left of $\Gamma_{0}$. However, for our purposes, we assume that $\widehat{\zeta}(s)$ may be continued analytically in an appropriate way; we shall want to take for $\Gamma_{0}$ a deformed contour $\Gamma$ in $\Sigma_{\phi}^{\vartheta}=\{0\} \cup\{s \neq 0: 1$ $\operatorname{args} \mid<\phi\}$, which has asymptotic behavior in the left complex plane, with Res $\rightarrow-\infty$ when Ims $\rightarrow \pm \infty$, which force $e^{s t}$ to decay towards both ends of $\Gamma$. In our work, we choose $\Gamma$ as

$$
s(\gamma)=\vartheta+\rho-\rho \sin (\eta-\imath \gamma)), \quad \gamma \in \mathbb{R},
$$

where

$$
\begin{gathered}
0<\eta<\phi-\frac{\pi}{2}, \\
\rho>0, \\
\vartheta>0 .
\end{gathered}
$$

Letting $s=\varsigma+\iota$, we see that (15) represents the left branch of the hyperbola given by

$$
\left(\frac{\varsigma-\vartheta-\rho}{\rho \sin \eta}\right)^{2}-\left(\frac{\zeta}{\rho \cos \eta}\right)^{2}=1,
$$

where $\zeta= \pm(\varsigma-\vartheta-\rho) \cot \eta$ are the asymptotes for (17) and $s=\vartheta+\rho(1-\sin \eta)$ is its $x$-intercept. It is confirmed from Eq. (16) that $\Gamma$ lies in the sector $\Sigma^{\vartheta}{ }_{\varphi}=\vartheta+\Sigma_{\varphi} \subset \Sigma_{\varphi}$ and grows into the left half plane. From (15) and (14), we get

$$
\zeta(t)=\frac{1}{2 \pi \iota} \int_{-\infty}^{\infty} e^{s(\gamma) \tau} \widehat{\zeta}(s(\gamma)) s^{\prime}(\gamma) d \gamma
$$

The approximation of Eq. (18) using the trapezoidal rule with uniform step $k$ is given as

$\zeta_{k}(t)=\frac{k}{2 \pi \iota} \sum_{j=-N}^{N} e^{s_{j} t} \widehat{\zeta}\left(s_{j}\right) s_{j}^{\prime}, \quad \gamma_{j}=j k, s_{j}=s\left(\gamma_{j}\right), s_{j}^{\prime}=s^{\prime}\left(\gamma_{j}\right)$.

3.1. Error Analysis. While solving the fractional-order problem defined in Eqs. (1)-(3), the first step is the application of Laplace transform to the given problem. The Laplace transform reduces the problem to a time-independent problem in Laplace space, and in this process, no error occurs. Next, the problem is solved in complex space for the unknown, and this process also incurs no error. In the final step, the solution of the given problem is retrieved using the Laplace inverse. We represent our solution as integral (18). We approximate this integral by the trapezoidal rule. During this process, integral (18) converges at different time rates which depends on $\Gamma$. In the process of numerical approximation of the Bromwich integral (18), the orders of convergence greatly depend on the step $k$ of the trapezoidal rule and the temporal domain $\left[t_{0}, T\right]$. For useful results and strong convergence, a suitable temporal domain is chosen. The next theorem gives the order of quadrature error.

Theorem 5 (see [47], Theorem 2.1). Let $\zeta(t)$ be the solution of (1) with $\widehat{\zeta}(s)$ analytic in $\Sigma^{\vartheta}{ }_{\varphi}$. Let $\Gamma \subset \Omega_{r} \subset \Sigma^{\vartheta}{ }_{\varphi}$ and define $\cosh (b)=\left(\theta \tau_{1} \sin (\eta)\right)^{-1}$, for $b>0$, where $0<\tau_{0}<T, \tau_{1}=$ $t_{0} / T, 0<\theta<1.0$, and let $\beta=\theta \bar{r} N / b T$. Then, for Eq. (19), with $k=b / N \leq \bar{r} / \log 2$, we have $\left|\zeta(t)-\zeta_{k}(t)\right| \leq C Q e^{9 \tau_{1}} l\left(\left\|\zeta_{0}\right\|+\| \hat{\mathscr{H}}\right.$ $\left.\|_{\Sigma_{\phi}^{9}}\right)\left(\rho_{r} N\right) e^{-\mu N}$, for $\mu=\bar{r}(1-\theta) / b, \rho_{r}=\theta \bar{r} \tau_{1} \sin \left(\eta-r_{1}\right) / b, \bar{r}$ $=2 \pi r_{1}, r_{1}>0, \tau_{0} \leq t \leq T, l(x)=\max (1, \log (1 / x))$, and $C=$ $C_{\eta, r_{1}, \beta_{1}}$. Hence, we have

$$
\text { error }_{\text {est }}=\left|\zeta_{k}(t)-\zeta(t)\right|=O\left(e^{-\mu N}\right) l\left(\rho_{r} N\right)
$$

\section{Stehfest Method (SM)}

In the Stehfest method, the approximate value of $\zeta(t)$ is given as

$$
\zeta(t)=\frac{\ln 2}{t} \sum_{i=1}^{M} w_{i} \widehat{\zeta}\left(\frac{\ln 2}{t} i\right)
$$

where the weights $w_{i}$ are given by

$$
w_{i}=(-1)^{M / 2+i} \sum_{h=\lfloor(i+1) / 2\rfloor}^{\min (i, M / 2)} \frac{h^{M / 2}(2 h !)}{(M / 2-h) ! h !(h-1) !(i-h) !(2 h-i) !} .
$$

Solving (12) for the corresponding Laplace parameters $s$ $=(\ln 2 / t) i, i=1,2,3, \cdots, M$.

The solution of the original problem can be obtained using (21).

4.1. Error Analysis. The authors [48, 49] performed a large number of numerical experiments to study the effect of the parameter on numerical accuracy, and they summarized there experimental work as the following: "If $j$ significant digits are desired, then let $M$ be the positive integer $\lceil 1.1 j\rceil$. Given $M$, set the system precision at $\lceil 2.2 M\rceil$. Given $M$ and the system precision, calculate the weights $w_{i}, 1 \leq i \leq 2 M$, using (22). Then, given the transform $\widehat{\zeta}(s)$ and the argument 
TABLe 1: The approximate solutions for various values of $\beta$ and $t \in[0,1]$ corresponding to Problem 1 .

\begin{tabular}{|c|c|c|c|c|c|c|c|c|}
\hline \multirow{2}{*}{$t$} & \multicolumn{2}{|c|}{$\beta=1$} & \multicolumn{2}{|c|}{$\beta=0.9$} & \multicolumn{2}{|c|}{$\beta=0.8$} & \multicolumn{2}{|c|}{$\beta=0.7$} \\
\hline & CIM & SM & CIM & SM & CIM & SM & CIM & SM \\
\hline 0.1 & 0.0998 & 0.0998 & 0.2171 & 0.2171 & 0.3349 & 0.3349 & 0.4519 & 0.4519 \\
\hline 0.2 & 0.1987 & 0.1987 & 0.3163 & 0.3163 & 0.4312 & 0.4312 & 0.5416 & 0.5416 \\
\hline 0.3 & 0.2955 & 0.2955 & 0.4070 & 0.4070 & 0.5131 & 0.5131 & 0.6118 & 0.6118 \\
\hline 0.4 & 0.3894 & 0.3894 & 0.4905 & 0.4905 & 0.5839 & 0.5839 & 0.6680 & 0.6680 \\
\hline 0.5 & 0.4794 & 0.4794 & 0.5668 & 0.5668 & 0.6447 & 0.6447 & 0.7120 & 0.7120 \\
\hline 0.6 & 0.5646 & 0.5646 & 0.6355 & 0.6355 & 0.6959 & 0.6959 & 0.7450 & 0.7450 \\
\hline 0.7 & 0.6442 & 0.6442 & 0.6965 & 0.6965 & 0.7376 & 0.7376 & 0.7673 & 0.7673 \\
\hline 0.8 & 0.7174 & 0.7174 & 0.7492 & 0.7492 & 0.7698 & 0.7698 & 0.7794 & 0.7794 \\
\hline 0.9 & 0.7833 & 0.7834 & 0.7934 & 0.7934 & 0.7925 & 0.7925 & 0.7816 & 0.7816 \\
\hline 1 & 0.8415 & 0.8415 & 0.8286 & 0.8287 & 0.8057 & 0.8057 & 0.7740 & 0.7740 \\
\hline
\end{tabular}

TABle 2: For various values of $\beta, N, M$ the absolute errors corresponding to Problem 1.

\begin{tabular}{|c|c|c|c|c|c|c|}
\hline \multirow{2}{*}{$(N, M)$} & \multicolumn{2}{|c|}{$\beta=1$} & \multicolumn{2}{|c|}{$\beta=0.9$} & \multicolumn{2}{|c|}{$\beta=0.8$} \\
\hline & CIM & SM & CIM & SM & CIM & SM \\
\hline$(30,6)$ & $3.19 \times 10^{-4}$ & $1.15 \times 10^{-1}$ & $1.03 \times 10^{-2}$ & $7.88 \times 10^{-2}$ & $3.10 \times 10^{-2}$ & $3.18 \times 10^{-2}$ \\
\hline$(60,8)$ & $5.21 \times 10^{-6}$ & $2.56 \times 10^{-2}$ & $1.28 \times 10^{-2}$ & $1.64 \times 10^{-2}$ & $3.58 \times 10^{-2}$ & $4.30 \times 10^{-3}$ \\
\hline$(90,10)$ & $4.25 \times 10^{-8}$ & $4.70 \times 10^{-3}$ & $1.28 \times 10^{-2}$ & $1.51 \times 10^{-2}$ & $3.58 \times 10^{-2}$ & $3.58 \times 10^{-2}$ \\
\hline$(120,12)$ & $6.69 \times 10^{-11}$ & $1.90 \times 10^{-3}$ & $1.28 \times 10^{-2}$ & $1.46 \times 10^{-2}$ & $3.58 \times 10^{-2}$ & $3.75 \times 10^{-2}$ \\
\hline$(150,14)$ & $7.99 \times 10^{-12}$ & $6.96 \times 10^{-5}$ & $1.28 \times 10^{-2}$ & $1.28 \times 10^{-2}$ & $3.58 \times 10^{-2}$ & $3.59 \times 10^{-2}$ \\
\hline$(180,16)$ & $1.13 \times 10^{-13}$ & $6.41 \times 10^{-5}$ & $1.28 \times 10^{-2}$ & $1.28 \times 10^{-2}$ & $3.58 \times 10^{-2}$ & $3.57 \times 10^{-2}$ \\
\hline$(210,18)$ & $2.68 \times 10^{-15}$ & $2.97 \times 10^{-7}$ & $1.28 \times 10^{-2}$ & $1.28 \times 10^{-2}$ & $3.58 \times 10^{-2}$ & $3.58 \times 10^{-2}$ \\
\hline$(240,20)$ & $2.55 \times 10^{-15}$ & $5.45 \times 10^{-6}$ & $1.28 \times 10^{-2}$ & $1.28 \times 10^{-2}$ & $3.58 \times 10^{-2}$ & $3.58 \times 10^{-2}$ \\
\hline
\end{tabular}

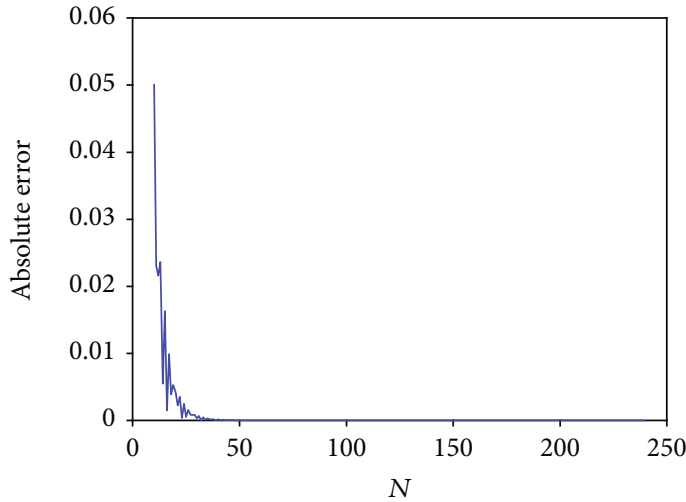

(a)

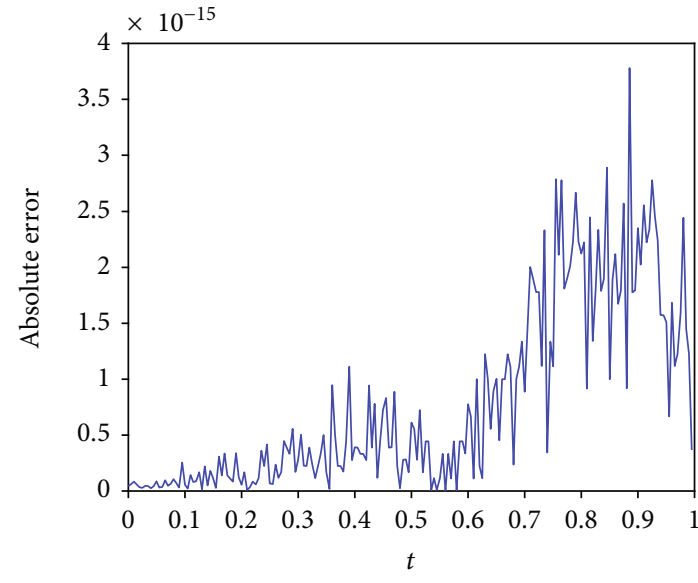

(b)

Figure 1: In (a), the error function for quadrature nodes $N$ and $\beta=1$ corresponding to Problem 1 is shown. In (b), the absolute error is shown for $\beta=1$; a very high accuracy is evident. 


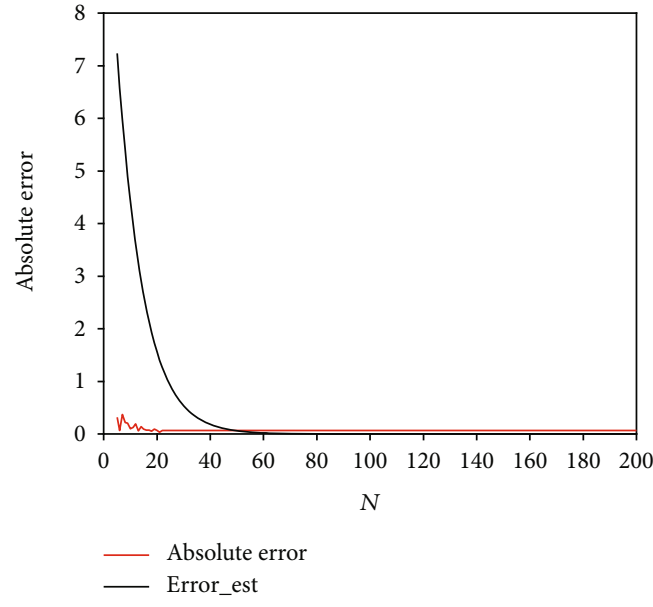

(a)

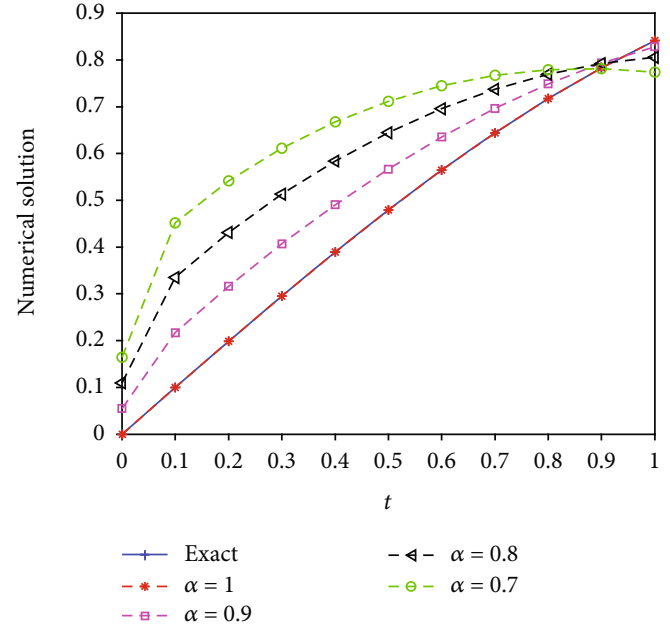

(b)

Figure 2: In (a), the absolute error versus error estimate corresponding to Problem 1 for $\beta=1$ is shown. From the figure, we see that the observed error agrees with the error estimate. In (b), the plots of approximate solutions for various $\beta$ are shown.

Table 3: The approximate solutions for various values of $\beta$ using the two numerical schemes corresponding to Problem 2.

\begin{tabular}{lcccccccc}
\hline$t$ & & $\beta=1$ & & \multicolumn{2}{c}{$\beta=0.9$} & \multicolumn{2}{c}{$\beta=0.8$} & \multicolumn{2}{c}{$\beta=0.7$} \\
\hline 0.1 & -0.0999 & -0.0999 & -0.2177 & -0.2177 & -0.3360 & -0.3360 & -0.4535 \\
0.2 & -0.1998 & -0.1998 & -0.3194 & -0.3194 & -0.4362 & -0.4362 & -0.5486 & -0.4535 \\
0.3 & -0.2993 & -0.2993 & -0.4149 & -0.4149 & -0.5251 & -0.5251 & -0.6279 & -0.6279 \\
0.4 & -0.3978 & -0.3978 & -0.5056 & -0.5056 & -0.6056 & -0.6056 & -0.6961 & -0.6961 \\
0.5 & -0.4947 & -0.4947 & -0.5914 & -0.5914 & -0.6785 & -0.6785 & -0.7547 & -0.7547 \\
0.6 & -0.5891 & -0.5891 & -0.6718 & -0.6718 & -0.7435 & -0.7435 & -0.8034 & -0.8034 \\
0.7 & -0.6799 & -0.6799 & -0.7457 & -0.7457 & -0.7997 & -0.7997 & -0.8416 & -0.8416 \\
0.8 & -0.7658 & -0.7658 & -0.8119 & -0.8119 & -0.8459 & -0.8459 & -0.8680 & -0.8680 \\
0.9 & -0.8453 & -0.8453 & -0.8690 & -0.8690 & -0.8808 & -0.8808 & -0.8812 & -0.8812 \\
1 & -0.9166 & -0.9166 & -0.9153 & -0.9153 & -0.9025 & -0.9025 & -0.8796 & -0.8796 \\
\hline
\end{tabular}

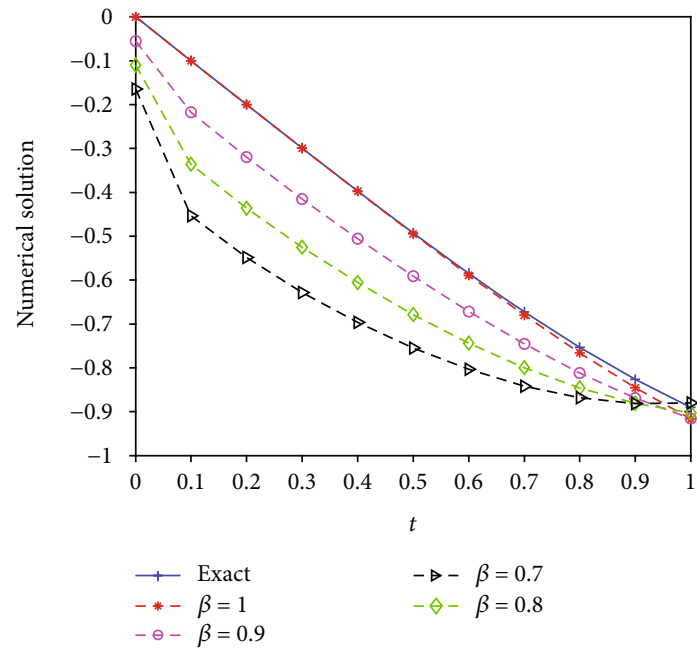

(a)

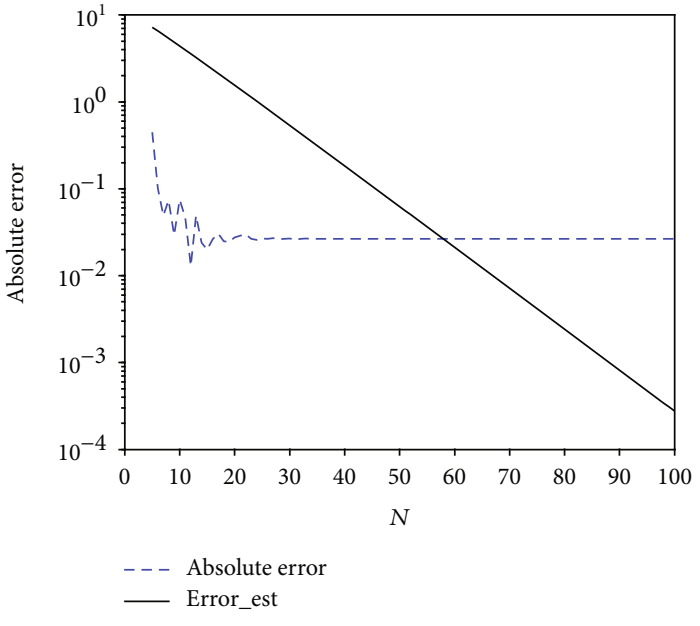

(b)

Figure 3: In (a), the graphs of numerical solutions for different values of $\beta$ corresponding to Problem 2 are shown. In (b), the absolute error vs. error estimates for $\beta=1$ are shown. 


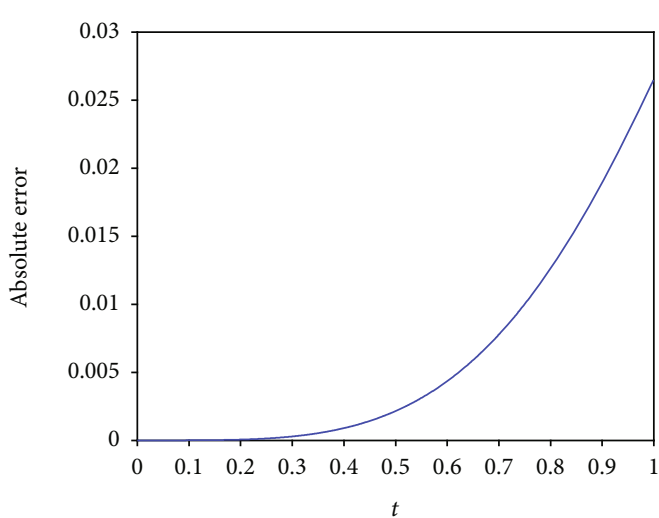

(a)

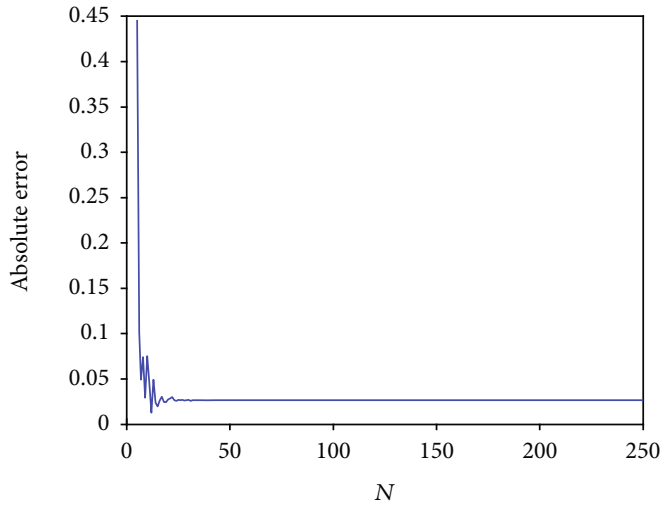

(b)

FIgure 4: In (a), absolute error versus $t$ for $\beta=1$ corresponding to Problem 2 is shown. In (b), the plot of error versus $N$ for $\beta=1$ is shown.

TABle 4: The approximate solutions for different values of $\beta$ using the two numerical schemes $N=240, M=22$, corresponding to Problem 3 .

\begin{tabular}{|c|c|c|c|c|c|c|c|c|}
\hline \multirow[b]{2}{*}{$t$} & \multicolumn{2}{|c|}{$\beta=1$} & \multicolumn{2}{|c|}{$\beta=0.9$} & \multicolumn{2}{|c|}{$\beta=0.8$} & \multicolumn{2}{|c|}{$\beta=0.7$} \\
\hline & CIM & SM & CIM & SM & CIM & SM & CIM & SM \\
\hline 0.1 & 1.1052 & 1.1054 & 1.2491 & 1.2501 & 1.4114 & 1.4113 & 1.5894 & 1.5917 \\
\hline 0.2 & 1.2214 & 1.2223 & 1.3893 & 1.3896 & 1.5744 & 1.5758 & 1.7719 & 1.7730 \\
\hline 0.3 & 1.3499 & 1.3506 & 1.5383 & 1.5398 & 1.7426 & 1.7441 & 1.9558 & 1.9571 \\
\hline 0.4 & 1.4918 & 1.4929 & 1.6994 & 1.6996 & 1.9213 & 1.9226 & 2.1492 & 2.1496 \\
\hline 0.5 & 1.6487 & 1.6494 & 1.8749 & 1.8751 & 2.1139 & 2.1157 & 2.3560 & 2.3575 \\
\hline 0.6 & 1.8221 & 1.8223 & 2.0667 & 2.0678 & 2.3229 & 2.3245 & 2.5795 & 2.5795 \\
\hline 0.7 & 2.0138 & 2.0145 & 2.2771 & 2.2781 & 2.5508 & 2.5520 & 2.8226 & 2.8241 \\
\hline 0.8 & 2.2255 & 2.2261 & 2.5082 & 2.5086 & 2.8003 & 2.8014 & 3.0880 & 3.0888 \\
\hline 0.9 & 2.4596 & 2.4599 & 2.7623 & 2.7633 & 3.0738 & 3.0753 & 3.3786 & 3.3770 \\
\hline 1 & 2.7183 & 2.7183 & 3.0422 & 3.0420 & 3.3742 & 3.3743 & 3.6974 & 3.6982 \\
\hline
\end{tabular}

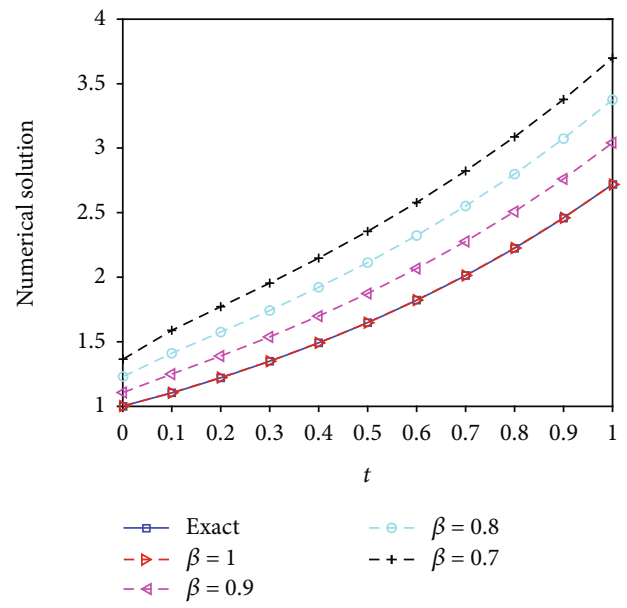

(a)

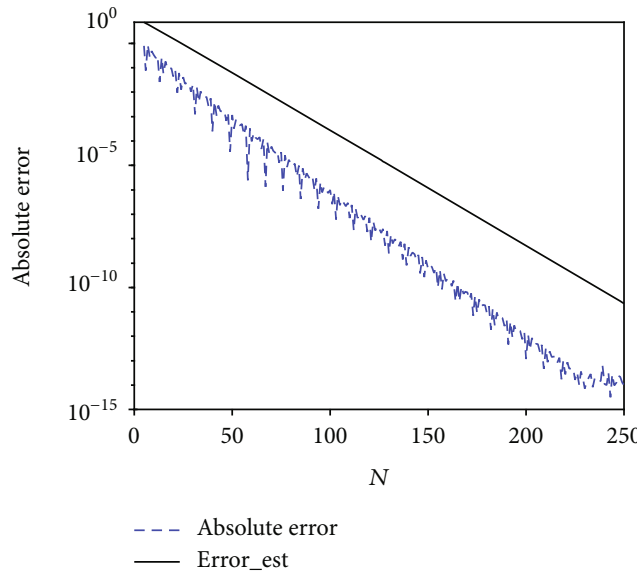

(b)

Figure 5: In (a), the numerical solutions for various values of $\beta$ corresponding to Problem 3 are shown. In (b), absolute error vs. error estimates for $\beta=1$ are shown. 


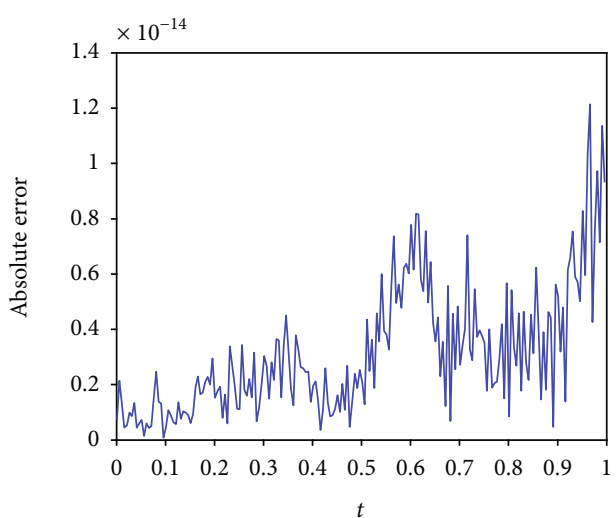

(a)

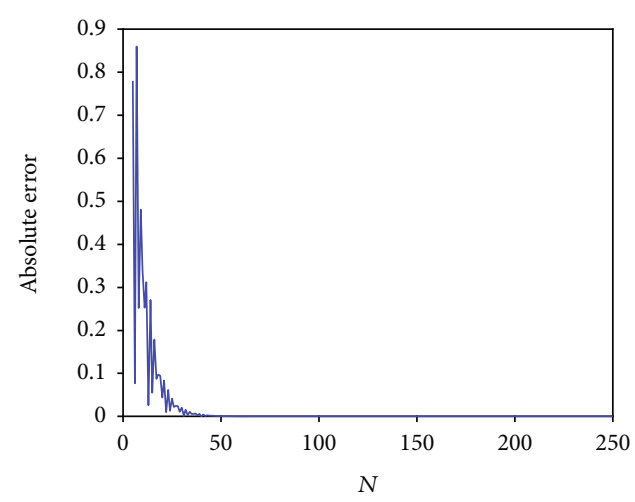

(b)

Figure 6: In (a), the error function for $t \in[0,1]$ and $\beta=1$ and in (b), for different quadrature nodes $N$ and $\beta=1$ for Problem 3 are shown.

TABLE 5: The approximate solutions of $\zeta_{1}$ for various values of $\beta$ using the two numerical schemes $N=240, M=22$, corresponding to Problem 4

\begin{tabular}{lcccccccc}
\hline & \multicolumn{3}{c}{$\beta=1$} & \multicolumn{2}{c}{$\beta=0.9$} & \multicolumn{2}{c}{$\beta=0.8$} & \multicolumn{2}{c}{$\beta=0.7$} \\
& CIM & SM & CIM & SM & CIM & SM & CIM & SM \\
\hline 0.1 & 0.1 & 0.1 & 0.2312 & 0.2313 & 0.3789 & 0.3791 & 0.5406 & 0.5409 \\
0.2 & 0.2 & 0.2 & 0.3395 & 0.3395 & 0.4926 & 0.4927 & 0.6550 & 0.6554 \\
0.3 & 0.3 & 0.3 & 0.4422 & 0.4422 & 0.5950 & 0.5953 & 0.7526 & 0.7529 \\
0.4 & 0.4 & 0.4 & 0.5415 & 0.5416 & 0.6906 & 0.6910 & 0.8408 & 0.8408 \\
0.5 & 0.5 & 0.5 & 0.6384 & 0.6385 & 0.7815 & 0.7818 & 0.9225 & 0.9228 \\
0.6 & 0.6 & 0.6 & 0.7333 & 0.7332 & 0.8688 & 0.8687 & 0.9994 & 0.9996 \\
0.7 & 0.7 & 0.7 & 0.8266 & 0.8267 & 0.9533 & 0.9534 & 1.0726 & 1.0730 \\
0.8 & 0.8 & 0.8 & 0.9186 & 0.9187 & 1.0354 & 1.0353 & 1.1426 & 1.1431 \\
0.9 & 0.9 & 0.9 & 1.0095 & 1.0096 & 1.1154 & 1.1158 & 1.2101 & 1.2102 \\
1 & 1 & 1 & 1.0993 & 1.0995 & 1.1936 & 1.1939 & 1.2754 & 1.2756 \\
\hline
\end{tabular}

$t$, calculate $\zeta(t)$ in (21)." According to these conclusions, the error is $10^{-j+1} \leq(\widehat{\zeta}-\zeta) / \zeta \leq 10^{-j}$, where $M=\lceil 1.1 j\rceil[50]$.

\section{Results and Discussion}

In order to check the accuracy and efficiency of the two numerical schemes. We consider linear and nonlinear and system of fractional-order VIDEs. In our numerical computations, the accuracy is achieved using the optimal parameters. The optimal values of the parameters utilized for CIM in our work are $\theta=0.1, \tau=t_{0} / T, \eta=0.15410, \bar{r}=2 \pi r, r=$ $0.13870,\left[t_{0}, T\right]=[0.5,5], \vartheta=2.0$. The MATLAB command $\gamma$ $=-N: k: N$ is used to generate the quadrature nodes for CIM. The parameter used in our numerical computation for the Stehfest method is $M$.

5.1. Problem 1. Here, we consider the fractional linear Volterra integrodifferential equation [31]:

$$
{ }_{0}^{\mathrm{ABC}} D_{t}^{\beta} \zeta(t)=1-\int_{0}^{t} \zeta(\tau) d \tau, t, \beta \in[0,1] .
$$

TABLE 6: The approximate solutions of $\zeta_{2}$ for various values of $\alpha$ using the two numerical schemes $N=240, M=20$, corresponding to Problem 4

\begin{tabular}{lcccccccc}
\hline & \multicolumn{2}{c}{$\beta=1$} & \multicolumn{2}{c}{$\beta=0.9$} & \multicolumn{2}{c}{$\beta=0.8$} & \multicolumn{2}{c}{$\beta=0.7$} \\
$t$ & CIM & SM & CIM & SM & CIM & SM & CIM & SM \\
\hline 0.1 & 1.1 & 1.1 & 1.2312 & 1.2312 & 1.3789 & 1.3789 & 1.5406 & 1.5406 \\
0.2 & 1.2 & 1.2 & 1.3395 & 1.3395 & 1.4926 & 1.4926 & 1.6550 & 1.6549 \\
0.3 & 1.3 & 1.3 & 1.4422 & 1.4421 & 1.5950 & 1.5949 & 1.7526 & 1.7526 \\
0.4 & 1.4 & 1.4 & 1.5415 & 1.5415 & 1.6906 & 1.6906 & 1.8408 & 1.8408 \\
0.5 & 1.5 & 1.5 & 1.6384 & 1.6384 & 1.7815 & 1.7816 & 1.9225 & 1.9225 \\
0.6 & 1.6 & 1.6 & 1.7333 & 1.7333 & 1.8688 & 1.8688 & 1.9994 & 1.9995 \\
0.7 & 1.7 & 1.7 & 1.8266 & 1.8266 & 1.9533 & 1.9533 & 2.0726 & 2.0726 \\
0.8 & 1.8 & 1.8 & 1.9186 & 1.9186 & 2.0354 & 2.0353 & 2.1426 & 2.1427 \\
0.9 & 1.9 & 1.9 & 2.0095 & 2.0095 & 2.1154 & 2.1153 & 2.2101 & 2.2101 \\
1 & 2 & 2 & 2.0993 & 2.0993 & 2.1936 & 2.1937 & 2.2754 & 2.2754 \\
\hline
\end{tabular}

TABLE 7: Absolute errors for $\beta=1$ using the two numerical schemes for different values of $N$ and $M$ corresponding to Problem 4.

\begin{tabular}{lcccc}
\hline \multirow{2}{*}{$(N, M)$} & $\zeta_{1}$ CIM & $\zeta_{2}$ & $\zeta_{1}$ & $\zeta_{2}$ \\
\hline$(30,6)$ & $3.19 \times 10^{-4}$ & $2.06 \times 10^{-2}$ & $2.10 \times 10^{-3}$ & $2.10 \times 10^{-3}$ \\
$(60,8)$ & $5.21 \times 10^{-6}$ & $9.70 \times 10^{-5}$ & $1.54 \times 10^{-4}$ & $1.54 \times 10^{-4}$ \\
$(90,10)$ & $4.25 \times 10^{-8}$ & $2.60 \times 10^{-6}$ & $3.47 \times 10^{-5}$ & $3.47 \times 10^{-5}$ \\
$(120,12)$ & $6.69 \times 10^{-11}$ & $7.08 \times 10^{-8}$ & $9.62 \times 10^{-7}$ & $9.62 \times 10^{-7}$ \\
$(150,14)$ & $7.99 \times 10^{-12}$ & $5.92 \times 10^{-10}$ & $3.60 \times 10^{-7}$ & $3.63 \times 10^{-7}$ \\
$(180,16)$ & $1.13 \times 10^{-13}$ & $9.22 \times 10^{-12}$ & $4.49 \times 10^{-8}$ & $3.38 \times 10^{-8}$ \\
$(210,18)$ & $3.88 \times 10^{-15}$ & $3.38 \times 10^{-13}$ & $2.01 \times 10^{-7}$ & $3.88 \times 10^{-7}$ \\
$(240,20)$ & $1.00 \times 10^{-15}$ & $5.71 \times 10^{-15}$ & $1.07 \times 10^{-6}$ & $3.38 \times 10^{-5}$ \\
\hline
\end{tabular}

The exact solution of this problem is $\zeta(t)=\sin (t)$. In Table 1, the approximate solutions of $\zeta(t)=\sin (t)$ for various values of $\beta$ and $t \in[0,1]$ using the two proposed methods are presented, and in Table 2 , the absolute errors are shown. The plot of error function for various quadrature nodes $N$ is 


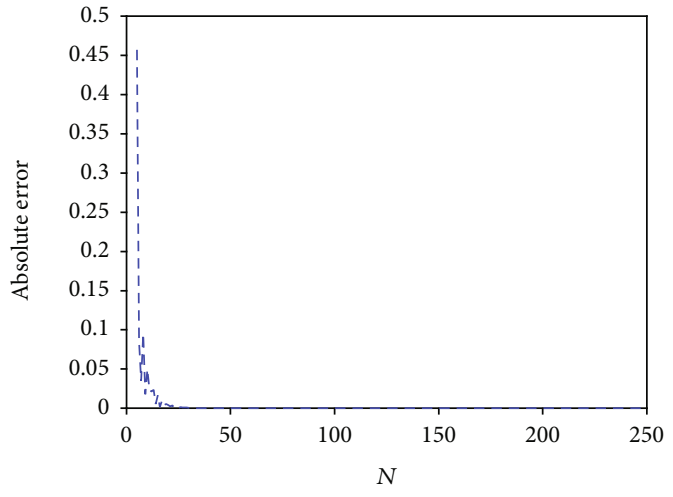

(a)

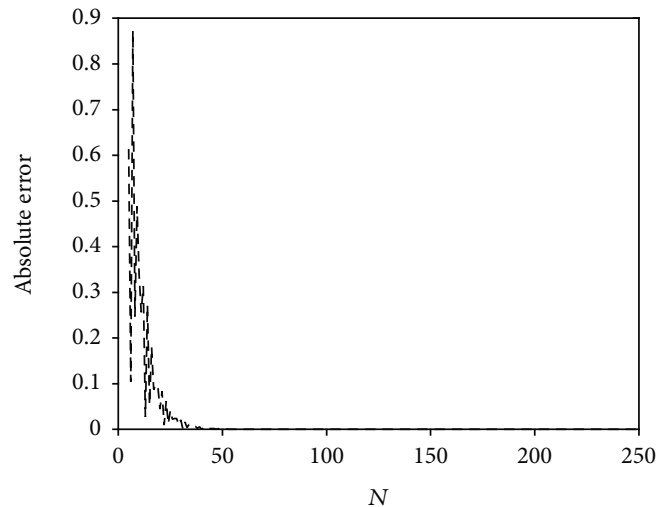

(b)

Figure 7: In (a), absolute error versus $N$ for fractional-order $\beta=1$ corresponding to Problem 4 for $\zeta_{1}$ is shown. In (b), the absolute error versus $N$ for $\beta=1$ corresponding to Problem 4 for $\zeta_{2}$ is shown.

shown in Figure 1(a), and the plot of the error function for $t \in[0,1]$ is shown in Figure 1(b). The graphs of approximate solutions for $\beta$ are presented in Figure 2(b), and Figure 2(a) displays the plot absolute error vs. error estimate for $\beta=1$. The numerical results using the Stehfest method are obtained by using $M=18$. All the plots are obtained using the CIM. It is evident that these methods can solve such type of equations efficiently.

5.2. Problem 2. Here, we consider the fractional nonlinear Volterra integrodifferential equation [31]:

$$
{ }_{0}^{\mathrm{ABC}} D_{t}^{\beta} \zeta(t)=-1+\int_{0}^{t} \zeta^{2}(\tau) d \tau, t, \beta \in[0,1]
$$

The problem has exact solution $\zeta(t)=\left((1 / 28) t^{4}-t\right)$ $\left((1 / 12) t^{3}+1\right)^{-1}$. The Stehfest parameter used is $M=18$. The numerical results for various values of $\beta$ and $t \in[0,1]$ are presented in Table 3 . The graphs of numerical solutions for various values of $\beta$ are shown in Figure 3(a), whereas Figure 3(b) presents the graph of error estimate vs. absolute error for $\beta=1$. For $t \in[0,1]$ and $\beta=1$, the error function is shown in Figure 4(a), and the error function for various $N$, and $\beta=1$ is shown in Figure 4(b). A similar performance is observed as was observed for Problem 1.

5.3. Problem 3. Here, we consider the fractional nonlinear Volterra integrodifferential equation [51]:

$$
\begin{aligned}
{ }_{0}^{\mathrm{ABC}} D_{t}^{\beta} \zeta(t)= & e^{t}\left(1+e^{t}\right)-t^{2} / 4\left(1-e^{2 t}+2 t e^{2 t}\right)-\zeta^{2}(t) \\
& +\int_{0}^{t} t^{2} \tau \zeta^{2}(\tau) d \tau, t, \beta \in[0,1] .
\end{aligned}
$$

The exact solution of this problem is $\zeta(t)=e^{t}$. The approximate solutions for different values of $\beta$ and $t \in[0,1]$ are presented in Table 4 . The Stehfest parameter used in this experiment is $M=22$. The graphs of approximate solutions for various values of $\beta$ are depicted in Figure 5(a), whereas Figure $3(\mathrm{~b})$ presents the plot of error estimate vs. observed error for $\beta=1$. In Figure 6(a), for $\beta=1$ and $t \in[0,1]$, the error function is shown, and in Figure 6(b) for different nodes $N$ and for $\beta=1$ is shown.

5.4. Problem 4. Here, we consider the fractional system of nonlinear Volterra integrodifferential equations [51]:

$$
\begin{gathered}
{ }_{0}^{\mathrm{ABC}} D_{t}^{\beta} \zeta_{1}(t)=1+t^{3}+t^{4}-\int_{0}^{t} t^{2}\left(\zeta_{1}(\tau)+\zeta_{2}(\tau)\right) d \tau, t, \beta \in[0,1] \\
D_{t}^{\beta} \zeta_{2}(t)=1-\frac{t^{3}}{2}-\frac{t^{4}}{3}+\int_{0}^{t} t \zeta_{1}(\tau) \zeta_{2}(\tau) d \tau, t, \beta \in[0,1] .
\end{gathered}
$$

The exact solutions of this system are $\zeta_{1}(t)=t$ and $\zeta_{2}(t)$ $=1+t$. The approximate solution of $\zeta_{1}(t)$ for various values of $\beta$ and $t \in[0,1]$ and $M=22$ is using the two numerical schemes $N=240, M=22$, corresponding to Problem 3 presented in Table 5, and the approximate solution of $\zeta_{2}(t)$ for various values of $\beta$ and $t \in[0,1]$ and $M=20$ is presented in Table 6 . In Table 7 , the errors obtained using the two proposed schemes are shown. For $\beta=1$ and various nodes $N$ for $\zeta 1(t)$, the error function is shown in Figure $7(\mathrm{a})$, and for $\zeta_{2}(t)$, the error function is shown in Figure $7(\mathrm{~b})$. The plots of numerical solutions of $\zeta_{1}(t)$ for different fractional-order $\beta$ are displayed in Figure 8(a), and the plots of numerical solutions of $\zeta_{2}(t)$ for various values of $\beta$ are displayed in Figure 8(b).

5.5. Problem 5. Here, we consider the fractional system of nonlinear Volterra integrodifferential equations [31]:

$$
\begin{aligned}
{ }_{0}^{\mathrm{ABC}} D_{t}^{\beta} \zeta_{1}(t)=1 & -\frac{\zeta_{1}^{2}(t)}{2}+\int_{0}^{t}\left((t-\tau) \zeta_{2}(\tau)\right. \\
& \left.+\zeta_{1}(\tau) \zeta_{2}(\tau)\right) d \tau, t, \tau, \beta \in[0,1] \\
D_{t}^{\beta} \zeta_{2}(t)=2 t & +\int_{0}^{t}\left((t-\tau) \zeta_{1}(\tau)-1\right) d \tau, t, \tau, \beta \in[0,1]
\end{aligned}
$$




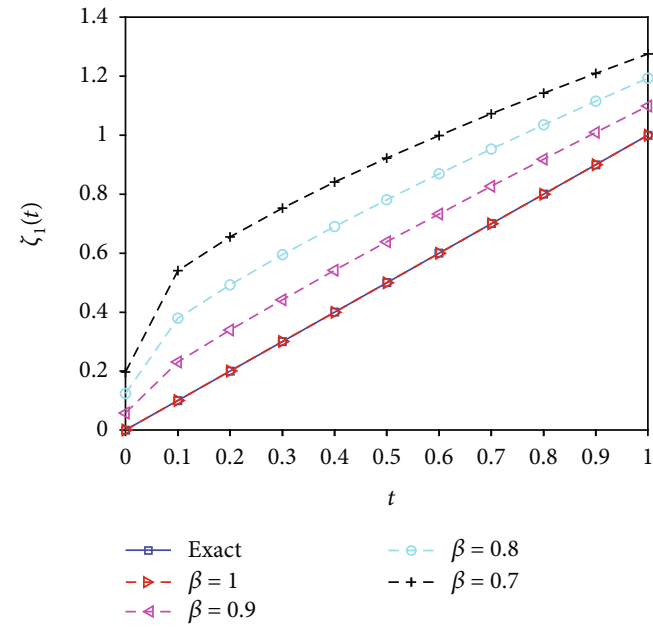

(a)

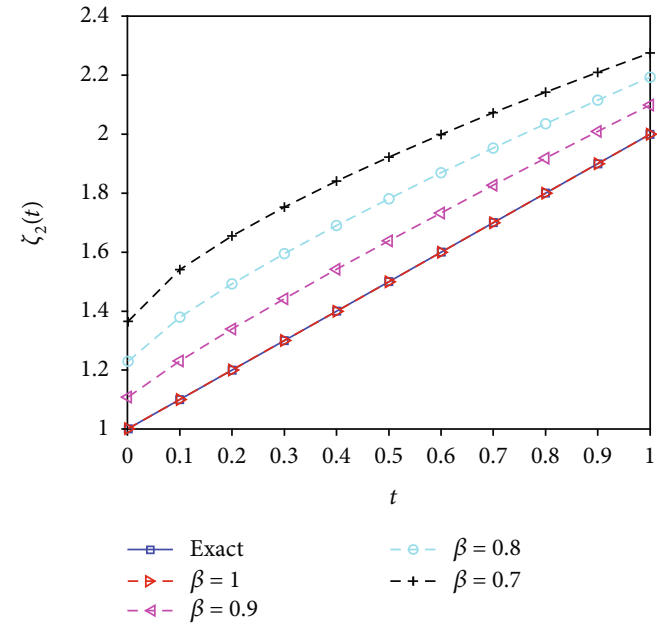

(b)

Figure 8: In (a), the numerical solutions corresponding to Problem 4 for fractional-order $\beta$ for $\zeta_{1}(t)$ are shown. In (b), the numerical solutions corresponding to Problem 4 for fractional-order $\beta$ for $\zeta_{2}(t)$ are shown.

TABLE 8: The approximate solutions for various values of $\beta$ using the CIM numerical schemes for $N=240$, corresponding to example 5 .

\begin{tabular}{|c|c|c|c|c|c|c|c|c|}
\hline \multirow{2}{*}{$t$} & \multicolumn{2}{|c|}{$\beta=1$} & \multicolumn{2}{|c|}{$\beta=0.9$} & \multicolumn{2}{|c|}{$\beta=0.8$} & \multicolumn{2}{|c|}{$\beta=0.7$} \\
\hline & $\zeta_{1}$ & $\zeta_{2}$ & $\zeta_{1}$ & $\zeta_{2}$ & $\zeta_{1}$ & $\zeta_{2}$ & $\zeta_{1}$ & $\zeta_{2}$ \\
\hline 0.1 & 0.1002 & 1.0050 & 0.2319 & 1.0172 & 0.3803 & 1.0311 & 0.5428 & 1.0466 \\
\hline 0.2 & 0.2013 & 1.0201 & 0.3433 & 1.0460 & 0.4993 & 1.0752 & 0.6648 & 1.1071 \\
\hline 0.3 & 0.3045 & 1.0453 & 0.4525 & 1.0858 & 0.6118 & 1.1308 & 0.7766 & 1.1792 \\
\hline 0.4 & 0.4108 & 1.0811 & 0.5629 & 1.1366 & 0.7238 & 1.1975 & 0.8868 & 1.2624 \\
\hline 0.5 & 0.5211 & 1.1276 & 0.6764 & 1.1985 & 0.8383 & 1.2756 & 0.9993 & 1.3567 \\
\hline 0.6 & 0.6367 & 1.1855 & 0.7947 & 1.2720 & 0.9575 & 1.3653 & 1.1171 & 1.4624 \\
\hline 0.7 & 0.7586 & 1.2552 & 0.9194 & 1.3576 & 1.0835 & 1.4673 & 1.2423 & 1.5803 \\
\hline 0.8 & 0.8881 & 1.3374 & 1.0520 & 1.4561 & 1.2179 & 1.5823 & 1.3768 & 1.7112 \\
\hline 0.9 & 1.0265 & 1.4331 & 1.1940 & 1.5684 & 1.3625 & 1.7113 & 1.5226 & 1.8561 \\
\hline 1 & 1.1752 & 1.5431 & 1.3469 & 1.6953 & 1.5190 & 1.8552 & 1.6813 & 2.0161 \\
\hline
\end{tabular}

TABle 9: The absolute error fractional-order $\beta=1$ and different quadrature nodes $N$ using the CIM numerical scheme, corresponding to Problem 5.

\begin{tabular}{lcc}
\hline$N$ & $\zeta_{1}$ & $\zeta_{2}$ \\
\hline 30 & $3.18 \times 10^{-4}$ & $2.03 \times 10^{-2}$ \\
60 & $5.21 \times 10^{-6}$ & $9.17 \times 10^{-5}$ \\
90 & $4.25 \times 10^{-8}$ & $2.61 \times 10^{-6}$ \\
120 & $6.69 \times 10^{-11}$ & $7.07 \times 10^{-8}$ \\
150 & $7.99 \times 10^{-12}$ & $5.84 \times 10^{-10}$ \\
180 & $1.13 \times 10^{-13}$ & $9.34 \times 10^{-12}$ \\
210 & $4.21 \times 10^{-15}$ & $3.40 \times 10^{-13}$ \\
240 & $1.99 \times 10^{-15}$ & $6.65 \times 10^{-15}$ \\
\hline
\end{tabular}

The exact solutions are $\zeta_{1}(t)=\sinh (t)$, and $\zeta_{2}(t)=\cosh$ $(t)$. The numerical solutions for various values of $\beta$ and $t \epsilon$ $[0,1]$ are presented in Table 8 .
The absolute errors for $\beta=1$, and quadrature nodes $N$ using the CIM are presented in Table 9. The plots of numerical solutions of $\zeta_{1}(t)$ for different fractional-orders $\beta$ are displayed in Figure 9(a), and the plots of numerical solutions of $\zeta_{2}(t)$ for various values $\beta$ are displayed in Figure 9(b). In Figure $10(\mathrm{a})$, the plots of error function of $\zeta_{1}(t)$ for $\beta=1$, and $t \in[0,1]$ are displayed, and in Figure 10(b), the plots of error function of $\zeta_{2}(t)$ for $\beta=1$, and $t \in[0,1]$ are displayed. The plots of error function of $\zeta_{1}(t)$ for various nodes $N$ and $\beta=1$ and are displayed in Figure 11(a), and the plots of error function of $\zeta_{2}(t)$ for quadrature nodes $N$ and $\beta=1$ are displayed in Figure 11(b).

\section{Conclusion}

In this work, we have successfully applied the Laplace transform to fractional VIDEs. The numerical inversion of Laplace transform is performed using the CIM and SM methods. In our numerical experiments, we considered linear, nonlinear, and system of fractional VIDEs with the 


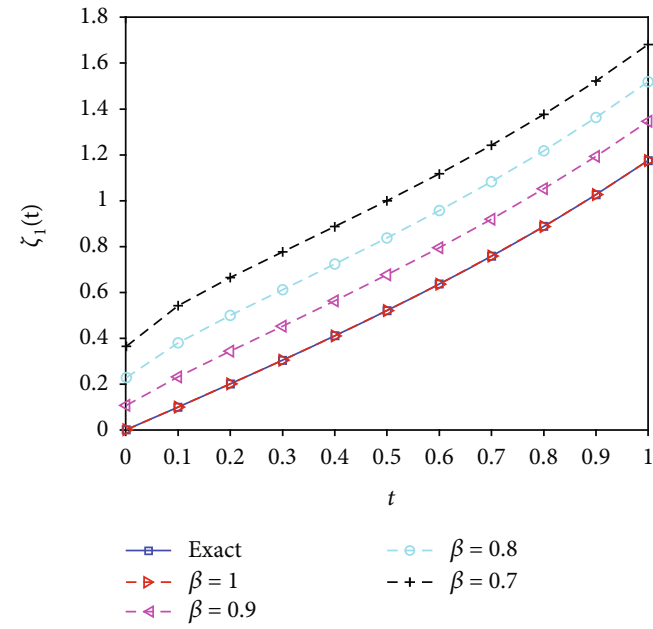

(a)

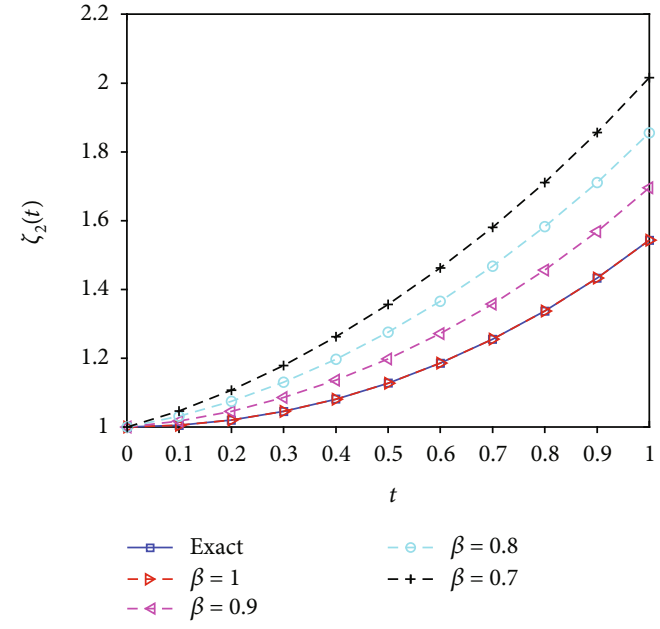

(b)

Figure 9: In (a), the numerical solutions corresponding to Problem 5 for fractional-order $\beta$ for $\zeta_{1}(t)$ are shown. In (b), the numerical solutions corresponding to Problem 5 for fractional-order $\beta$ for $\zeta_{2}(t)$ are shown.

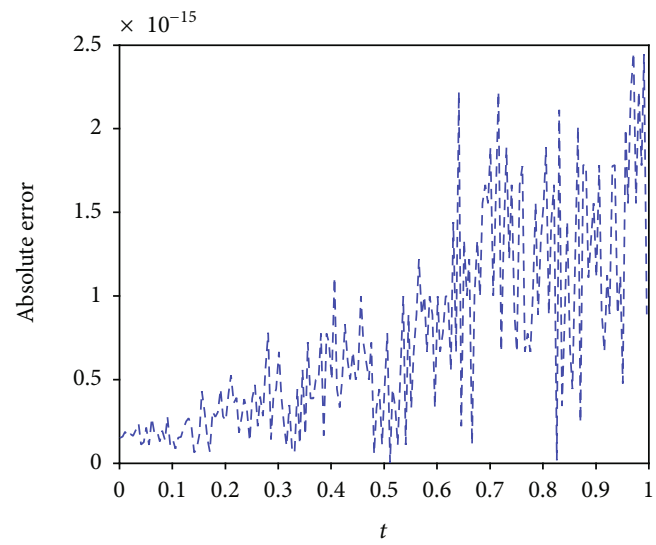

(a)

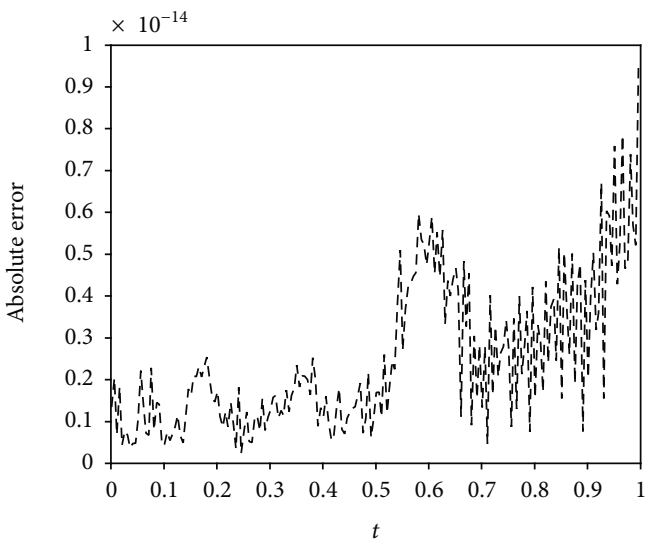

(b)

Figure 10: In (a), for $\beta=1$ and $t \in[0,1]$, the error function of $\zeta_{1}(t)$ corresponding to Problem 5 is shown. In (b), for $\beta=1$ and $t \in[0,1]$, the error function of $\zeta_{2}(t)$ corresponding to Problem 5 is shown.

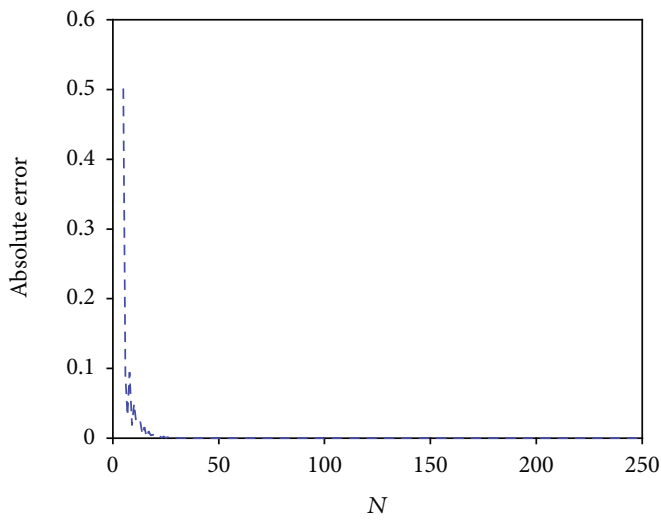

(a)

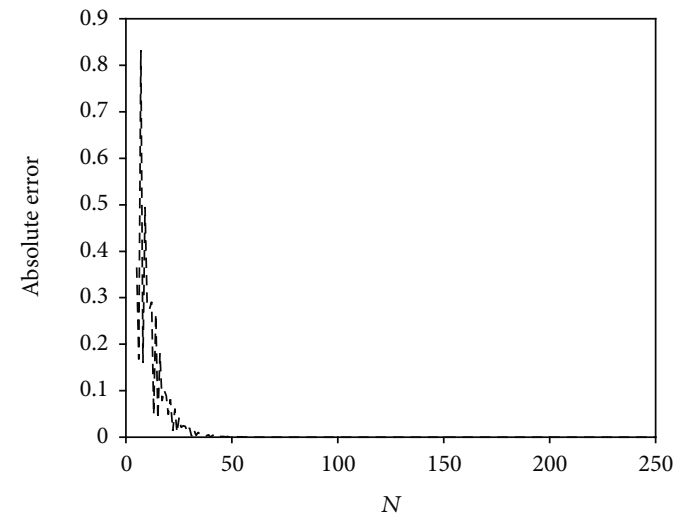

(b)

Figure 11: In (a), the plot shows various nodes $N$ along $\Gamma$ and $\beta=1$ the error function of $\zeta_{1}(t)$ corresponding to Problem 5. In (b), the plot shows various nodes $N$ along $\Gamma$ and $\beta=1$ the error function of $\zeta_{2}(t)$ corresponding to Problem 5. 
$\mathrm{ABC}$ derivative. The obtained results led us to the conclusion that the two proposed methods are capable of solving such type of equations. However, it was observed that the CIM is more accurate and converges faster than SM. Hence, the two proposed numerical schemes are the best alternative methods for solving such type of equations.

\section{Data Availability}

All data required for this paper is included within this paper.

\section{Conflicts of Interest}

The authors do not have any competing interests.

\section{Authors' Contributions}

Xiaoli Qiang wrote the paper, Kamran proved the results, Abid Mahboob revised the paper and arranged the funding, and Yu-Ming Chu supervised this work.

\section{Acknowledgments}

The research was supported by the National Natural Science Foundation of China (Grant Nos. 11971142, 11871202, $61673169,11701176,11626101$, and 11601485).

\section{References}

[1] Y. C. Kwun, G. Farid, W. Nazeer, S. Ullah, and S. M. Kang, "Generalized riemann-liouville $k$-fractional integrals associated with ostrowski type inequalities and error bounds of hadamard inequalities," IEEE Access, vol. 6, pp. 6494664953, 2018.

[2] W. Iqbal, K. M. Awan, A. U. Rehman, and G. Farid, “An extension of Petrovićs inequality for $h$-convex ( $h$-concave) functions in plane," Open Journal of Mathematical Sciences, vol. 3, no. 1, pp. 398-403, 2019.

[3] X. Yang, G. Farid, W. Nazeer, Y. M. Chu, and C. Dong, "Fractional generalized hadamard and Fejér-Hadamard inequalities for $<\mathrm{i}>\mathrm{m}</ \mathrm{i}>$-convex functions," AIMS Mathematics, vol. 5, no. 6, pp. 6325-6340, 2020.

[4] I. A. Baloch, S. S. Dragomir, and S. S. Dragomir, "New inequalities based on harmonic log-convex functions," Open Journal of Mathematical Analysis, vol. 3, no. 2, pp. 103-105, 2019.

[5] S. M. Kang, G. Farid, W. Nazeer, and B. Tariq, "Hadamard and Fejér-Hadamard inequalities for extended generalized fractional integrals involving special functions," Journal of Inequalities and Applications, vol. 2018, no. 1, 2018.

[6] R. L. Bagley and P. J. Torvik, "A theoretical basis for the application of fractional calculus to viscoelasticity," Journal of Rheology, vol. 27, no. 3, pp. 201-210, 1983.

[7] G. W. Bohannan, "Analog fractional order controller in temperature and motor control applications," Journal of Vibration and Control, vol. 14, no. 9-10, pp. 1487-1498, 2008.

[8] R. T. Baillie, "Long memory processes and fractional integration in econometrics," Journal of Econometrics, vol. 73, no. 1, pp. 5-59, 1996.

[9] M. Rahman, Integral Equations and Their Applications, WIT press, 2007.
[10] L. Debnath, "Recent applications of fractional calculus to science and engineering," International Journal of Mathematics and Mathematical Sciences, vol. 2003, no. 54, p. 3442, 2003.

[11] I. Podlubny, Fractional Differential Equations: An Introduction to Fractional Derivatives, Fractional Differential Equations, to Methods of their Solution and some of their Applications, Volume 198, Elsevier, 1998.

[12] S. Alkan and V. F. Hatipoglu, "Approximate solutions of Volterra-Fredholm integro-differential equations of fractional order," Tbilisi Mathematical Journal, vol. 10, no. 2, pp. 1-13, 2017.

[13] A. Saadatmandi and M. Dehghan, "A legendre collocation method for fractional integro-differential equations," Journal of Vibration and Control, vol. 17, no. 13, pp. 2050-2058, 2011.

[14] D. V. Bayram and A. Daşcıoğlu, "A method for fractional Volterra integro-differential equations by Laguerre polynomials," Advances in Difference Equations, vol. 2018, no. 1, 2018.

[15] D. Rani and V. Mishra, "Modification of Laplace adomian decomposition method for solving nonlinear volterra integral and integro-differential equations based on newton raphson formula," European Journal of Pure and Applied Mathematics, vol. 11, no. 1, pp. 202-214, 2018.

[16] Y. Nawaz, "Variational iteration method and homotopy perturbation method for fourth-order fractional integrodifferential equations," Computers \& Mathematics with Applications, vol. 61, no. 8, pp. 2330-2341, 2011.

[17] H. Ahmad, A. R. Seadawy, and T. A. Khan, "Study on numerical solution of dispersive water wave phenomena by using a reliable modification of variational iteration algorithm," Mathematics and Computers in Simulation, vol. 177, pp. 13-23, 2020.

[18] M. Rafiq, H. Ahmad, and S. T. Mohyud-Din, "Variational iteration method with an auxiliary parameter for solving Volterra's population model," Nonlinear Science Letters A, vol. 8, no. 4, pp. 389-396, 2017.

[19] O. A. Arqub and B. Maayah, "Numerical solutions of integrodifferential equations of Fredholm operator type in the sense of the Atangana-Baleanu fractional operator," Chaos, Solitons \& Fractals, vol. 117, pp. 117-124, 2018.

[20] V. E. Tarasov, "Fractional integro-differential equations for electromagnetic waves in dielectric media," Theoretical and Mathematical Physics, vol. 158, no. 3, pp. 355-359, 2009.

[21] R. P. Agarwal, B. de Andrade, and G. Siracusa, "On fractional integro-differential equations with state-dependent delay," Computers \& Mathematics with Applications, vol. 62, no. 3, pp. 1143-1149, 2011.

[22] S. Kermausuor, "Simpsons type inequalities for strongly (s, $\mathrm{m})$-convex functions in the second sense and applications," Open Journal of Mathematical Sciences, vol. 3, no. 1, pp. 7483, 2019.

[23] G. Farid, W. Nazeer, M. S. Saleem, S. Mehmood, and S. M. Kang, "Bounds of riemann-liouville fractional integrals in general form via convex functions and their applications," Mathematics, vol. 6, no. 11, p. 248, 2018.

[24] S. Mehmood, G. Farid, K. A. Khan, and M. Yussouf, "New hadamard and fej'er-hadamard fractional inequalities for exponentially m-convex function," Engineering and Applied Science Letters, vol. 3, pp. 45-55, 2020.

[25] W. Nazeer, S. M. Kang, M. Tanveer, and A. A. Shahid, "Fixed point results in the generation of Julia and Mandelbrot sets," Journal of Inequalities and Applications, vol. 2015, no. 1, Article ID 298, 2015. 
[26] M. Z. Sarikaya and S. Kaplan, "Some estimations ČebyševGrüss type inequalities involving functions and their derivatives," Open Journal of Mathematical Sciences, vol. 2, no. 1, pp. 146-155, 2018.

[27] A. Arikoglu and I. Ozkol, "Solution of fractional integrodifferential equations by using fractional differential transform method," Chaos, Solitons \& Fractals, vol. 40, no. 2, pp. 521529, 2009.

[28] E. A. Rawashdeh, "Legendre wavelets method for fractional integrodifferential equations," Applied Mathematical Sciences, vol. 5, no. 2, pp. 2467-2474, 2011.

[29] H. Jaradat, F. Awawdeh, and E. A. Rawashdeh, "Analytic solution of fractional integro-differential equations," Annals of the University of Craiova-Mathematics and Computer Science Series, vol. 38, no. 1, pp. 1-10, 2011.

[30] M. I. Syam, “Analytical solution of the fractional Fredholm integrodifferential equation using the fractional residual power series method," Complexity, vol. 2017, Article ID 4573589, 6 pages, 2017.

[31] O. A. Arqub and B. Maayah, "Fitted fractional reproducing kernel algorithm for the numerical solutions of ABC - fractional Volterra integro-differential equations," Chaos, Solitons \& Fractals, vol. 126, pp. 394-402, 2019.

[32] X. Ma and C. Huang, "Numerical solution of fractional integro-differential equations by a hybrid collocation method," Applied Mathematics and Computation, vol. 219, no. 12, pp. 6750-6760, 2013.

[33] O. A. Agbolade and T. A. Anake, "Solutions of first-order Volterra type linear integrodifferential equations by collocation method," Journal of Applied Mathematics, vol. 2017, Article ID 1510267, 5 pages, 2017.

[34] D. S. H. Mohammed, "Numerical solution of fractional integro-differential equations by least squares method and shifted Chebyshev polynomial," Mathematical Problems in Engineering, vol. 2014, Article ID 431965, 5 pages, 2014.

[35] A. M. Mahdy and R. T. Shwayyea, "Numerical solution of fractional integro-differential equations by least squares method and shifted Laguerre polynomials pseudo-spectral method," International Journal of Scientific \& Engineering Research, vol. 7, no. 4, pp. 1589-1596, 2016.

[36] N. Rajagopal, S. Balaji, R. Seethalakshmi, and V. S. Balaji, “A new numerical method for fractional order Volterra integrodifferential equations," Ain Shams Engineering Journal, vol. 11, no. 1, pp. 171-177, 2020.

[37] C. Yang and J. Hou, "Numerical solution of integrodifferential equations of fractional order by Laplace decomposition method," WSEAS Transactions on Mathematics, vol. 12, no. 12, pp. 1173-1183, 2013.

[38] F. Rabiei, F. A. Hamid, Z. A. Majid, and F. Ismail, "Numerical solutions of Volterra integro-differential equations using general linear method," Numerical Algebra, Control \& Optimization, vol. 9, no. 4, p. 433, 2019.

[39] A. A. Kilbas, H. M. Srivastava, and J. J. Trujillo, Theory and Applications of Fractional Differential Equations, Volume 204, Elsevier Science Limited, 2006.

[40] J. Hristov, "On the Atangana-Baleanu derivative and its relation to the fading memory concept: the diffusion equation formulation," in Fractional Derivatives with Mittag-Leffler Kernel. Studies in Systems, Decision and Control, J. Gómez, L. Torres, and R. Escobar, Eds., vol. 194, pp. 175-193, Springer, Cham, 2019.
[41] H. Bai, M. S. Saleem, W. Nazeer, M. S. Zahoor, and T. Zhao, "Hermite-Hadamard- and Jensen-type inequalities for intervalh1,h2nonconvex function," Journal of Mathematics, vol. 2020, Article ID 3945384, 6 pages, 2020.

[42] H. U. R. I. Y. E. Kadakal, M. A. H. I. R. Kadakal, and I. M. D. A. T. Iscan, "New type integral inequalities for three times differentiable preinvex and prequasiinvex functions," Open Journal of Mathematical Analysis, vol. 2, no. 1, pp. 33-46, 2018.

[43] S. Zhao, S. I. Butt, W. Nazeer, J. Nasir, M. Umar, and Y. Liu, "Some Hermite-Jensen-Mercer type inequalities for $\mathrm{k}$ Caputo-fractional derivatives and related results," Advances in Difference Equations, vol. 2020, no. 1, Article ID 262, 2020.

[44] G. Farid, "Existence of an integral operator and its consequences in fractional and conformable integrals," Open Journal of Mathematical Sciences, vol. 3, no. 3, pp. 210-216, 2019.

[45] S. I. Butt, M. Nadeem, and G. Farid, "On caputo fractional derivatives via exponential $(s, m)$-convex functions," Engineering and Applied Science Letter, vol. 3, no. 2, pp. 32-39, 2020.

[46] A. Atangana and D. Baleanu, "New fractional derivatives with nonlocal and non-singular kernel: theory and application to heat transfer model," Thermal Science, vol. 20, pp. 763-769, 2016.

[47] W. McLean and V. Thomee, "Numerical solution via Laplace transforms of a fractional order evolution equation," Journal of Integral Equations and Applications, vol. 22, no. 1, pp. 5794, 2010.

[48] J. Abate and P. P. Valko, "Multi-precision Laplace transform inversion," International Journal for Numerical Methods in Engineering, vol. 60, no. 5, pp. 979-993, 2004.

[49] J. Abate and W. Whitt, "A unified framework for numerically inverting Laplace transforms," INFORMS Journal on Computing, vol. 18, no. 4, pp. 408-421, 2006.

[50] Z. J. Fu, W. Chen, and H. T. Yang, "Boundary particle method for Laplace transformed time fractional diffusion equations," Journal of Computational Physics, vol. 235, pp. 52-66, 2013.

[51] R. M. Ganji and H. Jafari, “A new approach for solving nonlinear Volterra integro-differential equations with mittag-leffler kernel," Proceedings of the Institute of Mathematics and Mechanics, National Academy of Sciences of Azerbaijan, vol. 46, pp. 144-158, 2020. 